\title{
Quality-of-life estimation by Polish and American inflammatory bowel diseases patients - pilot study
}

\author{
Ocena jakości życia u polskich i amerykańskich pacjentów z nieswoistymi \\ chorobami zapalnymi jelit
}

\author{
Ludwika Jakubowska-Burek ${ }^{1,2}$, Izabella Warmuz-Stangierska ${ }^{3}$, Elżbieta Kaczmarek ${ }^{4}$, Marcin A. Kucharski ${ }^{1,2}$, \\ Emilia Marcinkowska ${ }^{5}$, Włodzimierz Szczepaniak ${ }^{1}$, Dorota Mańkowska-Wierzbicka ${ }^{1}$, Marian Grzymisławski ${ }^{5}$, \\ Krzysztof Linke', Jerzy Sowiński³ ${ }^{3}$ Agnieszka Dobrowolska-Zachwieja ${ }^{1,2}$
1Department of Gastroenterology, Human Nutrition and Internal Diseases, Poznan University of Medical Sciences, Poland 2Laboratory of Nutrigenetics, Department of Gastroenterology, Human Nutrition and Internal Diseases, Poznan University of Medical Sciences, Poland
3Department of Endocrinology, Metabolism and Internal Diseases, Poznan University of Medical Sciences, Poland
${ }^{4}$ Department of Bioinformatics and Computational Biology, Poznan University of Medical Sciences, Poland
5Department of Internal, Metabolic and Dietetic Diseases, Poznan University of Medical Sciences, Poland

Przegląd Gastroenterologiczny 2011; 6 (6): 388-400 DOI: $10.5114 / p g .2011 .25995$

Key words: inflammatory bowel diseases, quality of life, Polish and American population.

Słowa kluczowe: nieswoiste choroby zapalne jelit, jakość życia, populacja polska i amerykańska.

\begin{abstract}
Address for correspondence: Ludwika Jakubowska-Burek MSc, Department of Gastroenterology, Human Nutrition and Internal Diseases, Poznan University of Medical Sciences, 49 Przybyszewskiego, 60-355 Poznań, phone: +48 618691343 , fax: +48 618691686 , e-mail: ludwika.jakubowska@gmail.com
\end{abstract}

\begin{abstract}
Introduction: Crohn's disease (CD) and ulcerative colitis (UC) belong to inflammatory bowel diseases (IBD). Symptoms that occur primarily in $C D$ patients not only affect the gastrointestinal tract (GI), but also the extra-intestinal area, as well as patients' emotional and physiological well-being.

Aim: The aim of the study was to find out if there are any differences between the Polish and American populations regarding the occurrence of symptoms and the way they are perceived by the patients.

Material and methods: A 45-question-long anonymous experimental questionnaire was created; it concerned the mental, physical, and social changes the patients underwent. The investigated group consisted of 18 Polish and 16 American CD patients. The results were then compared to a similar group of healthy individuals (16 Polish and 12 Americans).

Results: Significant differences were observed among the two investigated populations. The American group, for example, did not suffer from any eye problems, while the Polish group did. On the other hand, the Polish group did not observe any skin changes, while Americans presented skin lesions, e.g. ulcerations of the skin, furuncles, abscesses, and lichen planus. Discrepancies in dermatological, rheumatological, gastroenterological, psychological and general health questions were also observed.
\end{abstract}

\section{Streszczenie}

Wstęp: Choroba Leśniowskiego-Crohna (ChLC) i wrzodziejące zapalenie jelita grubego (WZJG) należą do nieswoistych chorób zapalnych jelit (NChZJ). Objawy występujące u pacjentów dotkniętych tym schorzeniem dotyczą nie tylko przewodu pokarmowego. Znaczna część chorych uskarża się na objawy spoza przewodu pokarmowego oraz na problemy emocjonalne, psychologiczne i pogorszenie samopoczucia.

Cel: Określenie różnic w symptomatologii NChZJ oraz subiektywnych wyznaczników jakości życia osób z tym schorzeniem w populacji polskiej i amerykańskiej, z uwzględnieniem różnic kulturowych.

Materiat i metody: Na potrzeby niniejszego badania eksperymentalnie utworzono anonimowy kwestionariusz zawierający 45 pytań dotyczący jakości życia osób z NChzJ. Badania przeprowadzono w grupie 18 polskich i 16 amerykańskich pacjentów, a następnie porównano z wynikami z grupy kontrolnej, w skład której wchodziło 16 Polaków i 12 Amerykanów niechorujących na NChZJ. Badanie grupy kontrolnej miało na celu ustalenie ewentualnych różnic dotyczących ocen i postaw życiowych wynikających z odmienności kulturowych. W opracowaniu wyników oparto się na analizie jakościowej i procentowym określeniu częstości występowania dolegliwości. Wyniki: Uzyskane wyniki wskazywały na różnice między pacjentami polskimi i amerykańskimi w zakresie subiektyw- 
Conclusions: Polish patients were more content with their quality of life, although the symptoms they presented did not impair the daily life of either group of patients severely. In comparison to their Polish counterparts, the lives of American patients seemed to be affected by Crohn's disease to a greater extent, including their work and personal problems.

\section{Introduction}

Crohn's disease (CD) and ulcerative colitis (UC) are two disease entities which belong to inflammatory bowel diseases (IBD). The aetiology of these diseases remains uncertain, although it is believed that they are multifactorial, and the interactions between different immunological, environmental, as well as genetic factors play the key causative role.

Inflammatory bowel diseases are characterized by specific symptoms, mostly from the gastrointestinal tract, such as abdominal pain, loose stools often with blood, increased body temperature, increasing general weakness, loss of appetite and, in consequence, decrease in body weight. In the case of Crohn's disease, it is important to mention complications concerning the gastrointestinal tract, such as internal and external fistulas, as well as changes seen in and around the anal region including ulcerations, perianal abscesses and fistulas. Such changes may also involve the groin, vulva and scrotum, causing numerous therapeutic problems. Abscess formation is often accompanied by fever, erythema, tenderness and pain, which increases during defecation, sitting and walking.

Many IBD patients show symptoms from other organs and systems. Inflammation of the large joints knee, elbow, hip, ankle and wrist joints - is among the extra-intestinal symptoms; it is migratory in character, and usually lasts a few weeks. Moreover, arthritis of the ileo-sacral region and ankylosing spondylitis can progress continuously, despite the patient being treated for IBD. In some patients, liver and biliary system diseases are diagnosed, such as liver steatosis, primary sclerosing cholangitis, cholelithiasis and chronic active hepatitis. Iritis and scleritis belong to the most commonly diagnosed changes in the region of the eye. Additionally, deep vein thrombosis, pulmonary embolism, as well as thrombo-embolic vascular changes can occur as a result of the activation of clotting and thrombotic factors in IBD. Skin complications occur in the form of erythema nodosum and pyoderma gangrenosum. Finally, patients can complain about various other symptoms from other organs and systems which, in consequence, affect their nej oceny dotkliwości i lokalizacji objawów NChZJ oraz jakości życia.

Wnioski: Objawy choroby upośledzają codzienne życie polskich pacjentów z NChZJ odmiennie i w mniejszym stopniu niż życie pacjentów amerykańskich. Polscy pacjenci z NChZJ wydają się bardziej usatysfakcjonowani jakością życia w porównaniu z pacjentami amerykańskimi. Stwierdzono także rolę uwarunkowań kulturowych $w$ zróżnicowaniu subiektywnej oceny przebiegu choroby przez pacjentów polskich i amerykańskich.

everyday functioning, and modify their daily activities, as well as personal life plans [1-4].

Like many other diseases, IBD occurs in various populations; however, its incidence is increased in highly developed countries, such as the United States of America, Canada or Scandinavian countries. What can be observed in Europe is a characteristic gradient as far as the frequency of occurrence of this group of diseases is concerned: the closer to the equator, the lower the incidence rate. The differentiation concerns not only the frequency of occurrence per se, but also the predispositions for acquiring the disease, genetic background, as well as modes of treatment.

\section{Material and methods}

The question that arises is whether similar differentiation also concerns the perception of the disease by the patients with IBD themselves, including their subjective feelings and the influence the disease has on the quality of their life. Since the possibility arose to compare two culturally different patient populations - Poles and Americans - the decision was made to conduct research with the aim to analyse possible differences between these groups with respect to the quality of everyday life of subjects with IBD [5].

The aim of the study was to find out if there are any subjective differences in evaluating the symptoms, as well as markers of the quality of life, in Polish and American IBD populations, also taking into consideration the cultural differences. This paper is the result of a pilot study, which is to be extended by examining subjects from the Asian population in the near future.

The participants were a group of 18 Polish and 16 American patients, whose results were compared to those of a control group, composed of 16 Poles and 12 Americans not affected with IBD. Healthy subjects from both populations (Polish and American) were included in the study in order to prevent falsification of the results due to cultural differences. The patients were enrolled in the research as part of the proceedings of the American students' scholarly circle, and were volunteers. Since the students had the opportunity to come into contact with patients from two different popula- 
tions, they examined the subjects in the Department of Gastroenterology, Human Nutrition and Internal Diseases at the Poznan University of Medical Sciences, as well as in the USA as part of a summer internship. All the investigated individuals agreed to take part in the study anonymously and to answer the questionnaire. Although we do realize that the research group is small, it is only a pilot group and it will be extended, both in terms of the populations analysed so far, and also an Asian population group shall be added.

The primary study method was an experimentally developed questionnaire, which analysed how the studied individuals subjectively assess their physical and psychological symptoms, as well as their functioning in everyday life. The questionnaire consisted of closed questions, chosen on the basis of personal clinical experiences, as well as data from the literature [6-9]; there were three possible answers to the questions: "yes", "no" and "don't know". The questions were arranged in such a way as to eliminate the suggestion component, as well as the so-called inner control. The questions concentrated on symptoms most commonly reported by the IBD patients concerning gastrointestinal, rheumatological, dermatological, ophthalmological, psychological symptoms, as well as general well-being and fitness. While compiling the results, only decidedly positive and negative answers were taken into consideration. The "don't know", overlooked, or contradictory answers were discarded.

Standardised research methods have not been applied, as we are planning to do so in a further stage of the study on a larger research group. In the initial stage we planned to use only a questionnaire created by the authors and based on the issues most frequently brought up in conversations with patients.

When compiling the obtained data, we focused on the quality analysis and percentage values of the frequency of occurrence. The results were presented in tables, which include the percentages and figures concerning the registered symptoms.

Statistical significance was evaluated using Fisher's exact test. Differences between the frequencies of occurrence were accepted as significant when $p<0.05$. Statistical analysis was performed using Statistica v. 8.0 (StatSoft Inc.)

The subject of the manuscript has been approved by the University School of Medical Sciences' Ethics Committee, number 385/08.

\section{Results}

The results pertaining to the frequency of occurrence are presented in the tables, which include the percentage values and figures concerning the registered symptoms (Table I-X). The analysis of the results was

Table I. Polish - gastroenterological questions

Tabela I. Populacja polska - pytania dotyczace dziedziny gastroenterologii

\begin{tabular}{|c|c|c|c|c|c|c|c|c|}
\hline Answer & $\begin{array}{c}\text { More } \\
\text { frequently } \\
\text { I see blood } \\
\text { in the stool }\end{array}$ & $\begin{array}{l}\text { Sometimes } \\
\text { even for } \\
\text { a few days } \\
\text { I am not able } \\
\text { to pass } \\
\text { a stool }\end{array}$ & $\begin{array}{l}\text { I often have } \\
\text { indigestion }\end{array}$ & $\begin{array}{l}\text { I often have } \\
\text { aphthous } \\
\text { lesions } \\
\text { in my mouth }\end{array}$ & $\begin{array}{l}\text { When I leave } \\
\text { my home } \\
\text { I have to } \\
\text { know the } \\
\text { localization } \\
\text { of the toilet } \\
\text { at the place } \\
\text { where } \\
\text { I am going }\end{array}$ & $\begin{array}{l}\text { I am } \\
\text { often } \\
\text { troubled } \\
\text { with } \\
\text { bloating } \\
\text { of the } \\
\text { abdomen }\end{array}$ & $\begin{array}{l}\text { Gastrointestinal } \\
\text { problems } \\
\text { and diseases } \\
\text { occurred } \\
\text { among the } \\
\text { relatives } \\
\text { on my } \\
\text { mother's side }\end{array}$ & $\begin{array}{c}\text { Because } \\
\text { of loose } \\
\text { stools } \\
\text { I spend } \\
\text { less time } \\
\text { outside } \\
\text { of home }\end{array}$ \\
\hline \multicolumn{9}{|c|}{ Controls } \\
\hline Yes & 0 & 3 & 3 & 0 & 1 & 4 & 2 & 0 \\
\hline No & 14 & 11 & 11 & 14 & 13 & 10 & 11 & 14 \\
\hline Yes $\%$ & 0.0 & 21.4 & 21.4 & 0.0 & 7.1 & 28.6 & 15.4 & 0.0 \\
\hline No\% & 100.0 & 78.6 & 78.6 & 100.0 & 92.9 & 71.4 & 84.6 & 100.0 \\
\hline \multicolumn{9}{|c|}{ IBD patients } \\
\hline Yes & 4 & 1 & 7 & 5 & 9 & 14 & 3 & 4 \\
\hline No & 14 & 16 & 11 & 10 & 14 & 4 & 12 & 11 \\
\hline Sum & 18 & 17 & 18 & 15 & 23 & 18 & 15 & 15 \\
\hline Yes\% & 22.2 & 5.9 & 38.9 & 33.3 & 39.1 & 77.8 & 20.0 & 26.7 \\
\hline No\% & 77.8 & 94.1 & 61.1 & 66.7 & 60.9 & 22.2 & 80.0 & 73.3 \\
\hline $\begin{array}{l}p \text {-Level } \\
\text { control vs. IBD }\end{array}$ & NS & NS & NS & $p<0.05$ & $p=0.056$ & $p=0.01$ & NS & NS \\
\hline
\end{tabular}


Table II. American - gastroenterological questions

Tabela II. Populacja amerykańska - pytania dotyczące dziedziny gastroenterologii

\begin{tabular}{|c|c|c|c|c|c|c|c|c|}
\hline Answer & $\begin{array}{c}\text { More } \\
\text { frequently } \\
\text { I see blood } \\
\text { in the stool }\end{array}$ & $\begin{array}{l}\text { Sometimes } \\
\text { even for } \\
\text { a few days } \\
\text { I am not able } \\
\text { to pass } \\
\text { a stool }\end{array}$ & $\begin{array}{l}\text { I often have } \\
\text { indigestion }\end{array}$ & $\begin{array}{l}\text { I often have } \\
\text { aphthous } \\
\text { lesions } \\
\text { in my mouth }\end{array}$ & $\begin{array}{l}\text { When I leave } \\
\text { my home } \\
\text { I have to } \\
\text { know the } \\
\text { localization } \\
\text { of the toilet } \\
\text { at the place } \\
\text { where } \\
\text { I am going }\end{array}$ & $\begin{array}{l}\text { I am } \\
\text { often } \\
\text { troubled } \\
\text { with } \\
\text { bloating } \\
\text { of the } \\
\text { abdomen }\end{array}$ & $\begin{array}{l}\text { Gastrointestinal } \\
\text { problems } \\
\text { and diseases } \\
\text { occurred } \\
\text { among the } \\
\text { relatives } \\
\text { on my } \\
\text { mother's side }\end{array}$ & $\begin{array}{c}\text { Because } \\
\text { of loose } \\
\text { stools } \\
\text { I spend } \\
\text { less time } \\
\text { outside } \\
\text { of home }\end{array}$ \\
\hline \multicolumn{9}{|c|}{ Controls } \\
\hline Yes & 0 & 2 & 2 & 0 & 1 & 1 & 2 & 0 \\
\hline No & 11 & 10 & 10 & 12 & 11 & 11 & 10 & 12 \\
\hline Yes\% & 0.0 & 16.7 & 16.7 & 0.0 & 8.3 & 8.3 & 16.7 & 0.0 \\
\hline No\% & 100.0 & 83.3 & 83.3 & 100.0 & 91.7 & 91.7 & 83.3 & 100.0 \\
\hline \multicolumn{9}{|c|}{ IBD patients } \\
\hline Yes & 11 & 2 & 5 & 5 & 14 & 13 & 6 & 12 \\
\hline No & 5 & 14 & 11 & 11 & 0 & 3 & 9 & 4 \\
\hline Sum & 16 & 16 & 16 & 16 & 14 & 16 & 15 & 16 \\
\hline Yes\% & 68.8 & 12.5 & 31.2 & 31.2 & 100.0 & 81.2 & 40.0 & 75.0 \\
\hline No\% & 31.2 & 87.5 & 68.8 & 68.8 & 0.0 & 18.8 & 60.0 & 25.0 \\
\hline $\begin{array}{l}p \text {-Level } \\
\text { control vs. IBD }\end{array}$ & $p<0.005$ & NS & NS & $p=0.053$ & $p<0.0001$ & $p<0.005$ & NS & $p=0.001$ \\
\hline
\end{tabular}

Table III. Polish - rheumatological questions

Tabela III. Populacja polska - pytania dotyczące dziedziny reumatologii

\begin{tabular}{|c|c|c|c|c|c|}
\hline Answer & $\begin{array}{l}\text { I have knee } \\
\text { joint pain which } \\
\text { causes me great } \\
\text { discomfort }\end{array}$ & $\begin{array}{l}\text { I have joint } \\
\text { pain which often } \\
\text { decreases my } \\
\text { mobility }\end{array}$ & $\begin{array}{l}\text { I have a very } \\
\text { troublesome back } \\
\text { and hip pain, } \\
\text { even when I am } \\
\text { lying down }\end{array}$ & $\begin{array}{l}\text { I often experience } \\
\text { stiffening of } \\
\text { my wrists, even } \\
\text { when I don't feel } \\
\text { any pain }\end{array}$ & $\begin{array}{l}\text { It often occurs } \\
\text { that my physical } \\
\text { ability is greatly } \\
\text { diminished, even } \\
\text { when I don't feel } \\
\text { joint and bone pain }\end{array}$ \\
\hline \multicolumn{6}{|c|}{ Controls } \\
\hline Yes & 0 & 0 & 2 & 0 & 1 \\
\hline No & 14 & 14 & 12 & 14 & 13 \\
\hline Yes $\%$ & 0 & 0 & 14.3 & 0 & 7.1 \\
\hline No\% & 100 & 100 & 85.7 & 100 & 92.9 \\
\hline \multicolumn{6}{|c|}{ IBD patients } \\
\hline Yes & 5 & 3 & 2 & 4 & 1 \\
\hline No & 13 & 13 & 15 & 14 & 16 \\
\hline \multicolumn{6}{|l|}{ Sum } \\
\hline Yes\% & 27.8 & 18.8 & 11.8 & 22.2 & 5.9 \\
\hline No\% & 72.2 & 81.2 & 88.2 & 77.8 & 94.1 \\
\hline $\begin{array}{l}p \text {-Level } \\
\text { control vs. IBD }\end{array}$ & $p=0.0525$ & NS & NS & NS & NS \\
\hline
\end{tabular}

based on the division into problematic areas concerning the organs affected by ailments, as well as different aspects of functioning in everyday life.
The analysis of the information from the questionnaires allowed us to pinpoint a number of discrepancies between the studied groups, which stemmed not 
Table IV. American - rheumatological questions

Tabela IV. Populacja amerykańska - pytania dotyczące dziedziny reumatologii

\begin{tabular}{|c|c|c|c|c|c|}
\hline Answer & $\begin{array}{l}\text { I have knee } \\
\text { joint pain which } \\
\text { causes me great } \\
\text { discomfort }\end{array}$ & $\begin{array}{l}\text { I have joint } \\
\text { pain which often } \\
\text { decreases my } \\
\text { mobility }\end{array}$ & $\begin{array}{l}\text { I have a very } \\
\text { troublesome back } \\
\text { and hip pain, } \\
\text { even when I am } \\
\text { lying down }\end{array}$ & $\begin{array}{l}\text { I often experience } \\
\text { stiffening of } \\
\text { my wrists, even } \\
\text { when I don't feel } \\
\text { any pain }\end{array}$ & $\begin{array}{l}\text { It often occurs } \\
\text { that my physical } \\
\text { ability is greatly } \\
\text { diminished, even } \\
\text { when I don't feel } \\
\text { joint and bone pain }\end{array}$ \\
\hline \multicolumn{6}{|c|}{ Controls } \\
\hline Yes & 3 & 2 & 0 & 0 & 2 \\
\hline No & 9 & 10 & 12 & 12 & 10 \\
\hline Yes $\%$ & 25.0 & 16.7 & 0.0 & 0.0 & 16.7 \\
\hline No\% & 75.0 & 83.3 & 100.0 & 100.0 & 83.3 \\
\hline \multicolumn{6}{|c|}{ IBD patients } \\
\hline Yes & 10 & 8 & 7 & 11 & 2 \\
\hline No & 6 & 8 & 9 & 5 & 14 \\
\hline \multicolumn{6}{|l|}{ Sum } \\
\hline Yes\% & 62.5 & 50.0 & 43.8 & 68.8 & 12.5 \\
\hline No\% & 37.5 & 50.0 & 56.2 & 31.2 & 87.5 \\
\hline $\begin{array}{l}p \text {-Level } \\
\text { control vs. IBD }\end{array}$ & $p=0.0671$ & NS & $p=0.570$ & $p=0.003$ & NS \\
\hline
\end{tabular}

only from the disease itself, but also from cultural differences.

In the group with gastroenterological symptoms predominating in IBD, but not noted in healthy individuals (and, therefore, independent of cultural differences), the following differences were observed:

- Polish patients complained of bloating more often (77.8\%, $p=0.01$ ), while American patients complained about the presence of blood in the stool $(68.8 \%$, $p<0.005)$;

- the most significant symptom, and at the same time a clear problem disturbing the everyday life of the American patients, was loose stools (75\%), as well as the constant need for access to toilet facilities (100\%); this problem applied to the Polish patients to a much smaller degree $(26.7 \%$ and $39 \%)$; the differences between the controls and IBD groups were more significant for the Americans (Table II) than for Polish patients (Table I).

Among the extra-intestinal symptoms the biggest differences concerned:

- ophthalmological symptoms - Americans more frequently reported problems with the eyes and reading difficulties $(37.5 \%, p=0.0237)$ than Polish patients (Table VII);

- rheumatological symptoms, in which there were differences in pain localization: Polish patients noted pain of the knee joints, and, as a result, limitations in physical abilities more often $(27.8 \%, p=0.0525)$ than the
Polish control group; however, for the Americans knee pain was not as significant ( $p=0.0671)$; the American patients, on the other hand, complained of wrist joints stiffening even if they do not feel the pain more frequently $(68.8 \%, p=0.003)$, as well as symptoms of back and hip pain (43.8\%, $p=0.057$ ) (Tables III and IV);

- in Polish patients, infections of the upper respiratory system and self-observation of depressive moods were not a particular problem $(p=0.068, p=0.0680$ respectively), while in the Americans, depression was a significant symptom ( $p=0.0390)$; the Americans also reported family history of gastrointestinal tract diseases on the father's side $(p=0.0393)$ (Tables IX and $\mathrm{X}$ ).

Differences seen despite cultural changes concerned: - tiredness - seen much more in American (58.3\% for control and $86.7 \%$ for IBD) than in Polish patients (4.3\% for control and $35.7 \%$ for IBD patients), $p=0.0023$ for IBD Polish and American patients, for controls - not significant;

- depression - present more frequently in the Polish population (healthy Polish individuals $35.7 \%$, healthy Americans 8.3\%), but escalated in IBD patients (Polish $55.6 \%$ and American 12.5\%), $p=0.0129$ for IBD Polish and American patients, for controls not significant;

- estimation of one's own body functioning - in the Polish group the disease was associated with a decrease of self-assessment (21.4\% of the healthy group of Polish individuals taking part in the questionnaire, and 


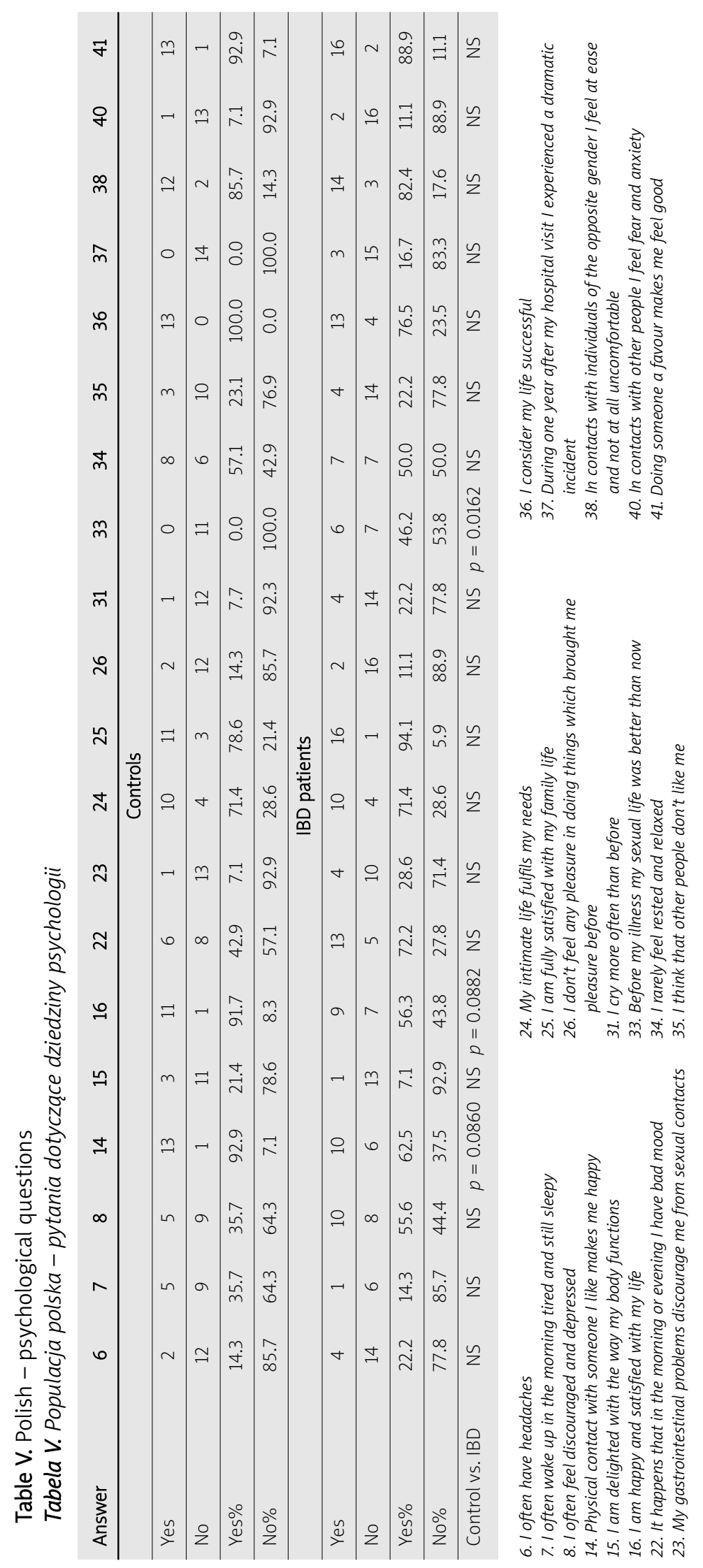




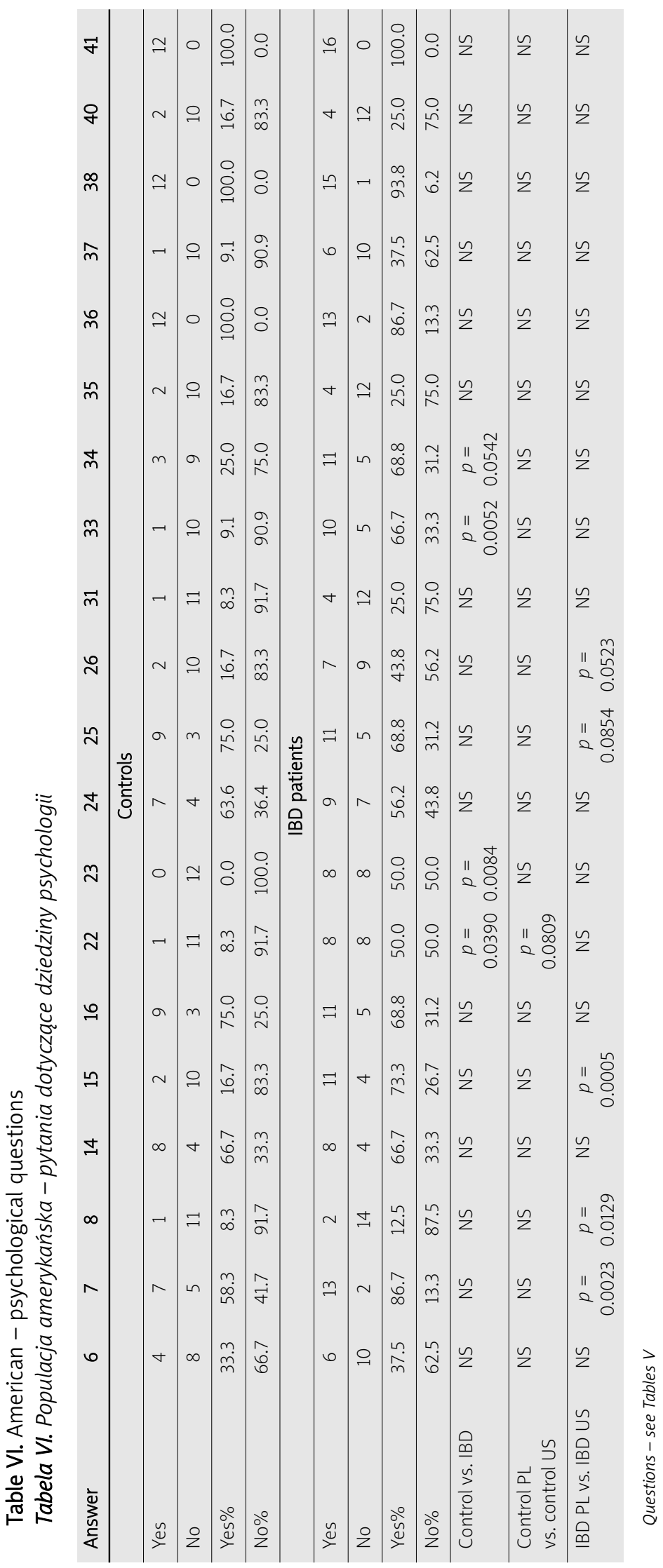


Table VII. Polish - questions about extra-intestinal symptoms

Tabela VII. Populacja polska - pytania o objawy spoza przewodu pokarmowego

\begin{tabular}{|c|c|c|c|c|c|}
\hline Answer & $\begin{array}{l}\text { I often get } \\
\text { blinded by the } \\
\text { light and have } \\
\text { trouble reading }\end{array}$ & $\begin{array}{l}\text { I frequently have } \\
\text { eye problems } \\
\text { (inflammation, } \\
\text { redness) }\end{array}$ & $\begin{array}{l}\text { I often have } \\
\text { a feeling of itching } \\
\text { under my eyelids, } \\
\text { have a feeling } \\
\text { of sand under } \\
\text { my eyelids }\end{array}$ & $\begin{array}{l}\text { Occasionally on my } \\
\text { extremities } \\
\text { appear red and } \\
\text { painful areas } \\
\text { (erythema } \\
\text { nodosum) }\end{array}$ & $\begin{array}{l}\text { I have observed } \\
\text { ulceration, } \\
\text { furuncles, } \\
\text { abscesses } \\
\text { or lichen } \\
\text { on my skin }\end{array}$ \\
\hline \multicolumn{6}{|c|}{ Controls } \\
\hline Yes & 4 & 3 & 1 & 0 & 0 \\
\hline No & 10 & 11 & 13 & 12 & 14 \\
\hline Yes\% & 28.6 & 21.4 & 7.1 & 0.0 & 0.0 \\
\hline No\% & 71.4 & 78.6 & 92.9 & 100.0 & 100.0 \\
\hline \multicolumn{6}{|c|}{ IBD patients } \\
\hline Yes & 3 & 5 & 3 & 2 & 6 \\
\hline No & 14 & 13 & 13 & 16 & 12 \\
\hline Sum & 17 & 18 & 16 & 18 & 18 \\
\hline Yes\% & 17.6 & 27.8 & 18.8 & 11.1 & 33.3 \\
\hline No\% & 82.4 & 72.2 & 81.3 & 88.9 & 66.7 \\
\hline$p$-Level control vs. IBD & NS & NS & NS & NS & NS \\
\hline
\end{tabular}

Table VIII. Americans - questions about extra-intestinal symptoms

Tabela VIII. Populacja amerykańska - pytania o objawy spoza przewodu pokarmowego

\begin{tabular}{|c|c|c|c|c|c|}
\hline Answer & $\begin{array}{l}\text { I often get } \\
\text { blinded by the } \\
\text { light and have } \\
\text { trouble reading }\end{array}$ & $\begin{array}{l}\text { I frequently have } \\
\text { eye problems } \\
\text { (inflammation, } \\
\text { redness) }\end{array}$ & $\begin{array}{l}\text { I often have } \\
\text { a feeling of itching } \\
\text { under my eyelids, } \\
\text { have a feeling } \\
\text { of sand under } \\
\text { my eyelids }\end{array}$ & $\begin{array}{l}\text { Occasionally on my } \\
\text { extremities } \\
\text { appear red and } \\
\text { painful areas } \\
\text { (erythema } \\
\text { nodosum) }\end{array}$ & $\begin{array}{l}\text { I have observed } \\
\text { ulceration, } \\
\text { furuncles, } \\
\text { abscesses } \\
\text { or lichen } \\
\text { on my skin }\end{array}$ \\
\hline \multicolumn{6}{|c|}{ Controls } \\
\hline Yes & 0 & 0 & 0 & 0 & 2 \\
\hline No & 12 & 12 & 12 & 12 & 10 \\
\hline Yes\% & 0.0 & 0.0 & 0.0 & 0.0 & 16.7 \\
\hline No\% & 100.0 & 100.0 & 100.0 & 100.0 & 83.3 \\
\hline \multicolumn{6}{|c|}{ IBD patients } \\
\hline Yes & 6 & 3 & 4 & 3 & 5 \\
\hline No & 10 & 12 & 12 & 13 & 11 \\
\hline Sum & 16 & 15 & 16 & 16 & 16 \\
\hline Yes\% & 37.5 & 20.0 & 25.0 & 18.8 & 31.2 \\
\hline No\% & 62.5 & 80.0 & 75.0 & 81.2 & 68.8 \\
\hline$p$-Level control vs. IBD & $p=0.0237$ & NS & NS & NS & NS \\
\hline
\end{tabular}

7.1\% among individuals affected by the disease), in the American group increase in self-assessment was observed $(16.7 \%$ healthy, and $73.3 \%$ among those affected by the disease), $p=0.0005$ for IBD Polish and American patients, for controls not significant;

- the feeling of good mood varied with little significance ( $p=0.0809$ ) even in American and Polish controls, whereas between IBD patients from the two investigated populations, the differences observed were not significant;

- the disease did not have a significant impact on the sexual functions in the group of American patients; on the other hand, it decreased the sexual satisfaction of Polish patients significantly (from $92.9 \%$ to $62.5 \%$, 
Table IX. Polish - general state of health questions

Tabela IX. Populacja polska - pytania o ogólny stan zdrowia

\begin{tabular}{|c|c|c|c|c|c|c|}
\hline Answer & $\begin{array}{l}\text { I often have } \\
\text { upper respiratory } \\
\text { infections } \\
\text { and get flu }\end{array}$ & $\begin{array}{c}\text { I have } \\
\text { problems } \\
\text { with my hair }\end{array}$ & $\begin{array}{l}\text { I observe } \\
\text { in myself signs } \\
\text { of depression }\end{array}$ & $\begin{array}{l}\text { From childhood } \\
\text { I suffered } \\
\text { from various } \\
\text { illnesses, } \\
\text { including } \\
\text { infectious ones }\end{array}$ & $\begin{array}{l}\text { I follow a very } \\
\text { regular } \\
\text { and hygienic } \\
\text { life style }\end{array}$ & $\begin{array}{l}\text { Gastrointestinal } \\
\text { tract diseases } \\
\text { occur on my } \\
\text { father's side } \\
\text { of the family }\end{array}$ \\
\hline \multicolumn{7}{|c|}{ Controls } \\
\hline Yes & 2 & 3 & 2 & 0 & 12 & 2 \\
\hline No & 12 & 11 & 12 & 14 & 2 & 11 \\
\hline Yes $\%$ & 14.3 & 21.4 & 14.3 & 0.0 & 85.7 & 15.4 \\
\hline No\% & 85.7 & 78.6 & 85.7 & 100.0 & 14.3 & 84.6 \\
\hline \multicolumn{7}{|c|}{ IBD patients } \\
\hline Yes & 8 & 6 & 8 & 1 & 13 & 3 \\
\hline No & 9 & 12 & 9 & 16 & 4 & 11 \\
\hline Sum & 17 & 18 & 17 & 17 & 17 & 14 \\
\hline Yes\% & 47.1 & 33.3 & 47.1 & 5.9 & 76.5 & 21.4 \\
\hline No\% & 52.9 & 66.7 & 52.9 & 94.1 & 23.5 & 78.6 \\
\hline $\begin{array}{l}p \text {-Level } \\
\text { control vs. IBD }\end{array}$ & $p=0.0680$ & NS & $p=0.0680$ & NS & NS & NS \\
\hline
\end{tabular}

Table X. American - general state of health questions

Tabela X. Populacja amerykańska - pytania o ogólny stan zdrowia

\begin{tabular}{|c|c|c|c|c|c|c|}
\hline Answer & $\begin{array}{l}\text { I often have } \\
\text { upper respiratory } \\
\text { infections } \\
\text { and get flu }\end{array}$ & $\begin{array}{l}\text { I have } \\
\text { problems } \\
\text { with my hair }\end{array}$ & $\begin{array}{l}\text { I observe } \\
\text { in myself signs } \\
\text { of depression }\end{array}$ & $\begin{array}{l}\text { From childhood } \\
\text { I suffered } \\
\text { from various } \\
\text { illnesses, } \\
\text { including } \\
\text { infectious ones }\end{array}$ & $\begin{array}{l}\text { I follow a very } \\
\text { regular } \\
\text { and hygienic } \\
\text { life style }\end{array}$ & $\begin{array}{l}\text { Gastrointestinal } \\
\text { tract diseases } \\
\text { occur on my } \\
\text { father's side } \\
\text { of the family }\end{array}$ \\
\hline \multicolumn{7}{|c|}{ Controls } \\
\hline Yes & 2 & 1 & 1 & 1 & 4 & 1 \\
\hline No & 10 & 11 & 11 & 11 & 8 & 11 \\
\hline Yes\% & 16.7 & 8.3 & 8.3 & 8.3 & 33.3 & 8.3 \\
\hline No\% & 83.3 & 91.7 & 91.7 & 91.7 & 66.7 & 91.7 \\
\hline \multicolumn{7}{|c|}{ IBD patients } \\
\hline Yes & 5 & 6 & 8 & 4 & 6 & 8 \\
\hline No & 11 & 9 & 8 & 11 & 9 & 8 \\
\hline Sum & 17 & 15 & 16 & 15 & 15 & 16 \\
\hline Yes\% & 29.4 & 40.0 & 50.0 & 26.7 & 40.0 & 50.0 \\
\hline No\% & 64.7 & 60.0 & 50.0 & 73.3 & 60.0 & 50.0 \\
\hline $\begin{array}{l}p \text {-Level } \\
\text { control vs. IBD }\end{array}$ & NS & $p=0.0914$ & $p=0.0390$ & NS & NS & $p=0.0390$ \\
\hline
\end{tabular}

$p=0.0860$ ); however, both populations of patients confirmed a decrease in the quality of sex life due to the disease (Polish patients $p=0.0162$, American patients $p=0.0052$ );

- in both populations the disease influenced social contacts with the opposite sex insignificantly, but it did shape family life very differently: in the Polish group (94.1\%) satisfaction gained from this area of life surpassed the satisfaction of healthy individuals (78.6\%); in the group of American patients the situation was opposite; healthy individuals experienced more satisfaction with family life $(p=0.0854)$; 
Table XI. Comparison of control and IBD patient groups as to the gastroenterological questions Tabela XI. Porównanie pacjentów z NChZJ i z grupy kontrolnej w zakresie pytań dotyczących dziedziny gastroenterologii

\begin{tabular}{|c|c|c|c|c|c|c|c|c|}
\hline & $\begin{array}{c}\text { More } \\
\text { frequently } \\
\text { I see blood } \\
\text { in the stool }\end{array}$ & $\begin{array}{l}\text { Sometimes } \\
\text { even for } \\
\text { a few days } \\
\text { I am not able } \\
\text { to pass } \\
\text { a stool }\end{array}$ & $\begin{array}{l}\text { I often have } \\
\text { indigestion }\end{array}$ & $\begin{array}{l}\text { I often have } \\
\text { aphthous } \\
\text { lesions } \\
\text { in my mouth }\end{array}$ & $\begin{array}{l}\text { When I leave } \\
\text { my home } \\
\text { I have to } \\
\text { know the } \\
\text { localization } \\
\text { of the toilet } \\
\text { at the place } \\
\text { where } \\
\text { I am going }\end{array}$ & $\begin{array}{l}\text { I am } \\
\text { often } \\
\text { troubled } \\
\text { with } \\
\text { bloating } \\
\text { of the } \\
\text { abdomen }\end{array}$ & $\begin{array}{l}\text { Gastrointestinal } \\
\text { problems } \\
\text { and diseases } \\
\text { occurred } \\
\text { among the } \\
\text { relatives } \\
\text { on my } \\
\text { mother's side }\end{array}$ & $\begin{array}{l}\text { Because } \\
\text { of loose } \\
\text { stools } \\
\text { I spend } \\
\text { less time } \\
\text { outside } \\
\text { of home }\end{array}$ \\
\hline $\begin{array}{l}p \text {-Level IBD PL } \\
\text { vs. IBD US }\end{array}$ & $p=0.0142$ & NS & NS & NS & $p=0.0002$ & NS & NS & $p=0.0121$ \\
\hline $\begin{array}{l}p \text {-Level } \\
\text { control PL } \\
\text { vs. control US }\end{array}$ & NS & NS & NS & NS & NS & NS & NS & NS \\
\hline
\end{tabular}

Table XII. Comparison of control and IBD patient groups as to the rheumatological questions Tabela XII. Porównanie pacjentów z NChZJ i z grupy kontrolnej w zakresie pytań dotyczących dziedziny reumatologii

\begin{tabular}{|c|c|c|c|c|c|}
\hline & $\begin{array}{l}\text { I have knee } \\
\text { joint pain which } \\
\text { causes me great } \\
\text { discomfort }\end{array}$ & $\begin{array}{l}\text { I have joint } \\
\text { pain which often } \\
\text { decreases my } \\
\text { mobility }\end{array}$ & $\begin{array}{l}\text { I have a very } \\
\text { troublesome back } \\
\text { and hip pain, } \\
\text { even when I am } \\
\text { lying down }\end{array}$ & $\begin{array}{l}\text { I often experience } \\
\text { stiffening of } \\
\text { my wrists, even } \\
\text { when I don't feel } \\
\text { any pain }\end{array}$ & $\begin{array}{l}\text { It often occurs } \\
\text { that my physical } \\
\text { ability is greatly } \\
\text { diminished, even } \\
\text { when I don't feel } \\
\text { joint and bone pain }\end{array}$ \\
\hline \multirow{2}{*}{$\begin{array}{l}p \text {-Level IBD PL } \\
\text { vs. IBD US }\end{array}$} & NS & NS & NS & NS & NS \\
\hline & $\begin{array}{l}\text { More frequently } \\
\text { I see blood } \\
\text { in the stool }\end{array}$ & $\begin{array}{l}\text { Sometimes even } \\
\text { for a few days } \\
\text { I am not able } \\
\text { to pass a stool }\end{array}$ & $\begin{array}{l}\text { I often have } \\
\text { indigestion }\end{array}$ & $\begin{array}{l}\text { I often have } \\
\text { aphthous } \\
\text { lesions } \\
\text { in my mouth }\end{array}$ & $\begin{array}{c}\text { When I leave } \\
\text { my home } \\
\text { I have to know } \\
\text { the localization } \\
\text { of the toilet at the place } \\
\text { where I am going }\end{array}$ \\
\hline $\begin{array}{l}p \text {-Level } \\
\text { control PL } \\
\text { vs. control US }\end{array}$ & $p=0.0824$ & NS & $p=0.0570$ & $p=0.0142$ & NS \\
\hline
\end{tabular}

Table XIII. Comparison of control and IBD patient groups as to the questions about extra-intestinal symptoms Tabela XIII. Porównanie pacjentów z NChZJ i z grupy kontrolnej w zakresie pytań o objawy spoza przewodu pokarmowego

\begin{tabular}{|c|c|c|c|c|c|}
\hline & $\begin{array}{l}\text { I often get blinded } \\
\text { by the light and have } \\
\text { trouble reading }\end{array}$ & $\begin{array}{l}\text { I frequently } \\
\text { have eye problems } \\
\text { (inflammation, } \\
\text { redness) }\end{array}$ & $\begin{array}{l}\text { I often have } \\
\text { a feeling of itching } \\
\text { under my eyelids, } \\
\text { have a feeling } \\
\text { of sand under my eyelids }\end{array}$ & $\begin{array}{l}\text { Occasionally on my } \\
\text { extremities appear } \\
\text { red and painful areas } \\
\text { (erythema nodosum) } \\
\text { s }\end{array}$ & $\begin{array}{l}\text { I have observed } \\
\text { ulceration, furuncles, } \\
\text { abscesses or lichen } \\
\text { on my skin }\end{array}$ \\
\hline \multicolumn{6}{|c|}{ Controls } \\
\hline $\begin{array}{l}p \text {-Level control } \\
\text { PL vs. control US }\end{array}$ & NS & NS & NS & NS & NS \\
\hline \multicolumn{6}{|c|}{ IBD patients } \\
\hline $\begin{array}{l}p \text {-Level IBD PL } \\
\text { vs. IBD US }\end{array}$ & NS & NS & NS & NS & NS \\
\hline
\end{tabular}


Table XIV. Comparison of control and IBD patient groups as to the general health state questions Tabela XIV. Porównanie pacjentów z NChZJ i z grupy kontrolnej w zakresie pytań o ogólny stan zdrowia

\begin{tabular}{|c|c|c|c|c|c|c|}
\hline & $\begin{array}{l}\text { I often have } \\
\text { ipper respiratory } \\
\text { infections } \\
\text { and get flu }\end{array}$ & $\begin{array}{l}\text { I have problems } \\
\text { with my hair }\end{array}$ & $\begin{array}{l}\text { I observe } \\
\text { in myself signs } \\
\text { of depression }\end{array}$ & $\begin{array}{l}\text { From childhood } \\
\text { I suffered } \\
\text { from various } \\
\text { illnesses, } \\
\text { including } \\
\text { infectious ones }\end{array}$ & $\begin{array}{l}\text { I follow a very } \\
\text { regular and } \\
\text { hygienic } \\
\text { life style }\end{array}$ & $\begin{array}{l}\text { Gastrointestinal } \\
\text { tract diseases } \\
\text { occur on my } \\
\text { father's side } \\
\text { of the family }\end{array}$ \\
\hline \multicolumn{7}{|c|}{ Controls } \\
\hline $\begin{array}{l}p \text {-Level control PL } \\
\text { vs. control US }\end{array}$ & NS & NS & NS & NS & $p=0.0138$ & NS \\
\hline \multicolumn{7}{|c|}{ IBD patients } \\
\hline $\begin{array}{l}p \text {-Level IBD PL } \\
\text { vs. IBD US }\end{array}$ & NS & NS & NS & NS & $p=0.0702$ & NS \\
\hline
\end{tabular}

- both American and Polish patients looked upon the onset of the disease as a stressful situation $(37.5 \%$ and $16.7 \%$, respectively), but the differences were not statistically significant.

Both patient populations displayed convergent opinions especially as far as psychosomatic symptoms were concerned:

- in both groups of patients symptoms of depression occurred more often than among healthy people (Tables IX and X);

- the disease did not increase the tendency to react by crying in either of the studied populations (Americans with $22 \%$ to $25 \%$, Poles with $7.7 \%$ to $8.3 \%$ ); however, patients from both populations reacted with fear in social situations and suffered from other diseases more frequently than healthy individuals;

- although patients from both populations observed a decrease in life satisfaction due to the disease (Poles with $91.7 \%$ to $56.3 \%, p=0.0882$, Americans with $75.0 \%$ to $68.8 \%$, NS), in the Polish group this was more prominent.

\section{Statistical analysis}

In the case of gastroenterological questions, in the group of American patients, issues concerning aphthous lesions in the mouth, as well as the question of leaving the house and being determined by the distribution of the toilet facilities on the way (also because of diarrhoea) were statistically significant (Tables I and II). This proves that American patients feel very restricted in their travel plans, or even during short walks from their home, by the availability of the sanitary infrastructure. This, in turn, considerably influences the quality of life, planning everyday activities, social meetings, as well as normal everyday functioning. In the Polish patients a similar tendency was observed, although it was not as intense as in the American IBD patient community. Addi- tionally, the presence of blood in the stool was observed with different frequencies in the Polish and American patients ( $p=0.0142)$, as well as a statistically significant need to know the location of toilets in the close vicinity $(p=0.0002)$, which restricted the time spent away from home $(p=0.0121)$ to a large extent.

In the group of questions devoted to rheumatological issues, the Americans reported greater problems caused by rheumatological symptoms; they complained of the discomfort caused by pain, or joint stiffness ( $p=0.0671)$, especially in the wrists $(p=0.003)$, as well as back and hip pain, even while lying down $(p=0.57)$. In the Polish group, only some statistically significant joint pain causing discomfort was recorded $(p=0.0525)$.

As far as the psychological questions were concerned, the Polish patients displayed a slight decrease in satisfaction gained from physical contact with their significant others $(p=0.086)$ (which was not noted in the American group at all), as well as a general fall of satisfaction with life ( $p=0.0882)$, which, in turn, was present in the case of the Americans ( $p=0.039$ ). Most of the patients, both Polish and American, also declared that before the disease had started their sexual life was much better (Polish $p=0.0162$, American $p=0.0052$ ). Americans furthermore seemed to feel relaxed and wellrested less often ( $p=0.0542$ ).

Among the questions pertaining to extra-intestinal symptoms, a statistically significant difference was noted in the case of the American patients, who, in comparison with the control group, complained about problems with reading and increased sensitivity to light much more frequently ( $p=0.0237)$. Nevertheless, no changes were observed in the frequency of occurrence of these problems between the control and patient groups.

The questions concerning the general state of health of those surveyed pointed to an increase with respect to the upper respiratory tract infections in the Polish group 
$(p=0.0680)$, who also presented a significantly higher percentage of mood depression $(p=0.0680)$. A similar tendency was observed in the results of the American group ( $p=0.0390)$. Moreover, the Americans complained of problems with their hair caused by the disease $(p=0.0914)$, and they reported gastroenterological problems in the family on the father's side $(p=0.0390)$. The perception of the subjects' own lifestyles differed as well: a statistically significant difference was observed both when comparing the two control groups $(p=0.0138)$, as well as the patients $(p=0.0702)$.

\section{Discussion}

The whole picture of a disease is comprised of the clinical symptoms verified by the laboratory findings, as well as the patients' subjective ailments and feelings. The latter make up an important link towards planning the diagnostic process and constitute a crucial therapeutic hint. A key element of treatment is to increase the quality of a patient's life; therefore, the therapy programme should include this aspect [10-14]. Consequently, the realization of the expectations and opinions of the patients constitutes an important factor of a physician's procedures. The data acquired from two different populations can make individual therapy easier, as well as adapt the treatment programme to the hierarchy of symptoms.

Despite the fact that the Polish group turned out as a whole (both IBD patients and healthy controls) to be more prone to react with a depressed mood, both patient groups presented substantially more depressive symptoms and tension, as well as less satisfaction with life in comparison to healthy individuals from both populations. This points to a clear connection between the disease and the emotional state of patients. In both patient populations an increase in reactions of fear and anxiety in social situations, along with a destructive influence of the disease in the sphere of sexual function, was also confirmed.

The perception of the symptoms and their influence on everyday life distinguished both populations from each other in many aspects. Unlike the Polish patients, in the American group dermatological symptoms were marginalized. Polish patients, on the other hand, did not mention any ophthalmological symptoms, while in the American group this was a significant factor decreasing their quality of life. Significant differences were also observed in the identification and localization of rheumatological, gastroenterological and psychological symptoms, as well as in the limitations imposed on everyday life. What was noted in the American group was paradoxically an increased assessment of one's own life-style, as well as body functioning despite the dise- ase, in comparison to the assessment of healthy individuals. Additionally, other diseases the patients had gone through, as well as family predispositions, were identified more frequently; also the feeling of fatigue appeared to be intensified more. It turns out that the disease affects the functioning of both populations in the sphere of social contacts differently: the feeling of rejection was dominant among the American patients, while in the Polish patient group the disease led to a decrease in socialising.

The disease also affected the family life of IBD patients differently - in the Polish group the disease strengthened family ties, whereas in the American group the situation was the opposite.

\section{Conclusions}

Inflammatory bowel diseases patients from both populations exhibited symptoms of tension, depression, lessened life satisfaction, as well as discomfort in social relations, which point to a huge emotional burden caused by the disease, decreasing the quality of their life significantly, though differently for each group.

Polish patients globally assessed the quality of their own life higher than the American patients did, and subjectively the symptoms of the disease hindered their everyday functioning to a lesser degree, which might suggest a better insight into their real needs and their feelings towards the disease.

The differences in the way Polish and American IBD patients function did not always result from the disease itself; cultural factors had a substantial influence on the overall picture as well. Differences in the symptoms expressed by the patients of both populations suggest the need for differentiation of the therapeutic approach (for instance, an increased emphasis on ophthalmological care in the American population, and on dermatological care in the Polish population).

Furthermore, psychotherapeutic care over the patients from both populations also requires a differentiated approach. In the American group, the problems of communication and family relations, as well as social acceptance, deserve more attention, whereas in the Polish group these are the issues of cooperation and a pro-social stance.

\section{Acknowledgments}

The research project was financed by the Department of Gastroenterology, Human Nutrition and Internal Diseases, Poznan University of Medical Sciences. The authors would like to thank the medical students Lukasz Janiak, Michael Losos and Natalia Pavelock for their time and effort in collecting material for this paper. Ludwika Jakubowska-Burek declares that she is a scholarship 
holder within the project "Scholarship support for PH.D. students specializing in majors strategic for Wielkopolska's development", Sub-measure 8.2.2 Human Capital Operational Programme, co-financed by European Union under the European Social Fund.

\section{References}

1. Subrata G, Rod M. Impact of inflammatory bowel disease on quality of life: results of the European Federation of Crohn's and Ulcerative Colitis Associations (EFCCA) patient survey. Aliment Pharmacol Ther 2002; 16: 1603-9.

2. Cohen RD. The quality of life in patients with Crohn's disease. J Crohn's Colitis 2007; 1: 10-20.

3. Steiner-Grossman P, Banks PA, Present DH, et al. The new people not patients: a source book for living with IBD. Dubuque, lowa: Kendall/Hunt Publishing Company, 1992.

4. Pace F, Molteni P, Bollani S, et al. Inflammatory bowel disease versus irritable bowel syndrome: a hospital-based, case-control study of disease impact on quality of life. Scand J Gastroenterol 2003; 38: 1031-8.

5. Price VE, Klaassen RJ, Bolton-Maggs P, et al. Measuring disease-specific quality of life in rare populations: a practical approach to cross-cultural translation. Health Qual Life Outcomes 2009; 7: 92.

6. Jordan-Marsh M. The SF-36 Quality-of-Life instrument: updates and strategies for critical care research. Critical Care Nurse 2002; 22: 35-43.

7. Chassany $\mathrm{O}$, Marquis P, Scherrer B, et al. Validation of a specific quality of life questionnaire for functional digestive disorders. Gut 1999; 44: 527-33.

8. Pallis AG, Mouzas IA. Instruments for quality of life assessment in patients with inflammatory bowel diseases. Digestiiuer Ois 2000; 32: 682-8.

9. Casellasa F, Lo'pez-Vivancosb J, Vergaraa M, et al. Impact of inflammatory bowel disease on health-related quality of life. Dig Dis 1999; 17: 208-18.

10. Afzal NA, Van der Zaag-Loonen HJ, Arnaud-Battandier F, et al. Improvement in quality of life of children with acute Crohn's disease does not parallel mucosal healing after treatment with exclusive enteral nutrition. Aliment Pharmacol Ther 2004; 20 167-72.

11. Gazzard BG. The quality of life in Crohn's disease. Gut 1987; 28: 378-81.

12. Broering DC, Eisenberger CF, Koch A, et al. Quality of life after surgical therapy of small bowel stenosis in Crohn's disease. Dig Surg 2001; 18: 124-30.

13. Papadakis KA. Adalimumab improves quality of life. Nat Rev Gastroenterol Hepatol 2009; 6: 200-1.

14. Irvine EJ, Greenberg GR, Feagan BG, et al. Quality of life rapidly improves with budesonide therapy for active Crohn's disease. Inflam J Bowel Dis 2000; 6: 181-7. 